Y. KUBOTA

KŌDAI MATH SEM REP.

28 (1977), 253-261

\title{
COEFFICIENTS OF MEROMORPHIC UNIVALENT FUNCTIONS
}

\author{
By Yoshinisa Kubota
}

1. We denote by $\Sigma^{\prime}$ the family of functions

$$
g(z)=z+\sum_{n=1}^{\infty} \frac{b_{n}}{z^{n}}
$$

regular and univalent in $1<|z|<\infty$. Let $g(z)$ be a function belonging to $\Sigma^{\prime}$ and let

$$
G(z)=z+\sum_{n=1}^{\infty} \frac{c_{n}}{z^{n}}
$$

be the inverse function of $g(z)$. The following results are known:

$$
\left|c_{1}\right|=\left|b_{1}\right| \leqq 1,\left|c_{2}\right|=\left|b_{2}\right| \leqq \frac{2}{3} .
$$

Springer [4] proved that $\left|c_{3}\right| \leqq 1$ and conjectured that

$$
\left|c_{2 n-1}\right| \leqq \frac{(2 n-2) !}{n !(n-1) !} \quad(n=1,2, \cdots) .
$$

In this paper we shall prove that the conjecture is true for the cases $n=3,4,5$.

THEOREM.

$$
\left|c_{5}\right| \leqq 2,\left|c_{7}\right| \leqq 5,\left|c_{9}\right| \leqq 14
$$

In these inequalities equality occurs only for the inverse function of $z+(1 / z)$ and its rotations.

Ozawa [3] made use of Grunsky's inequality together with Golusin's inequ. ality to prove the Bieberbach conjecture for the sixth coefficient. We apply his method to prove our theorem.

2. Let

$$
g(z)=z+\sum_{n=1}^{\infty} \frac{b_{n}}{z^{n}}
$$

be a function belonging to $\Sigma^{\prime}$ and let $F_{m}(w)$ be the $m$-th Faber polynomial Received Nov. 25, 1975. 
which is defined by

$$
g_{m}(z)=F_{m}[g(z)]=z^{m}+\sum_{n=1}^{\infty} \frac{a_{m n}}{z^{n}} .
$$

Then Grunsky's inequality has the form

$$
\left|\sum_{m, n=1}^{N} n a_{m n} x_{m} x_{n}\right| \leqq \sum_{n=1}^{N} n\left|x_{n}\right|^{2}
$$

and Golusin's inequality has the form

$$
\sum_{n=1}^{\infty} n\left|\sum_{m=1}^{N} x_{m} a_{m n}\right|^{2} \leqq \sum_{n=1}^{N} n\left|x_{n}\right|^{2}
$$

where $N$ is an arbitrary positive integer and $x_{1}, x_{2}, \cdots, x_{N}$ are arbitrary complex numbers.

By a simple calculation we have

$$
\begin{aligned}
a_{1 n}= & b_{n} \quad(n=1,2, \cdots), \\
a_{21}= & 2 b_{2}, \\
a_{22}= & 2 b_{3}+b_{1}{ }^{2}, \\
a_{23}= & 2 b_{4}+2 b_{1} b_{2}=\frac{2}{3} a_{32}, \\
a_{24}= & 2 b_{5}+2 b_{1} b_{3}+b_{2}{ }^{2}=\frac{1}{2} a_{42}, \\
a_{25}= & 2 b_{6}+2 b_{1} b_{4}+2 b_{2} b_{3}=\frac{2}{5} a_{52}, \\
a_{31}= & 3 b_{3}, \\
a_{33}= & 3 b_{5}+3 b_{1} b_{3}+3 b_{2}{ }^{2}+b_{1}{ }^{3}, \\
a_{35}= & 3 b_{7}+3 b_{1} b_{5}+6 b_{2} b_{4}+3 b_{3}{ }^{2}+3 b_{1}{ }^{2} b_{3}+3 b_{1} b_{2}{ }^{2}=\frac{3}{5} a_{53}, \\
a_{41}= & 4 b_{4}, \\
a_{44}= & 4 b_{7}+4 b_{1} b_{5}+8 b_{2} b_{4}+6 b_{3}{ }^{2}+4 b_{1}{ }^{2} b_{3}+8 b_{1} b_{2}{ }^{2}+b_{1}{ }^{4}, \\
a_{55}= & 5 b_{9}+5 b_{1} b_{7}+10 b_{2} b_{6}+15 b_{3} b_{5}+10 b_{4}{ }^{2}+5 b_{1}{ }^{2} b_{5}+20 b_{1} b_{2} b_{4}+15 b_{1} b_{3}{ }^{2}+20 b_{2}{ }^{2} b_{3} \\
& +5 b_{1}{ }^{3} b_{3}+15 b_{1}{ }^{2} b_{2}{ }^{2}+b_{1}{ }^{5} .
\end{aligned}
$$

Let

$$
g(z)=z+\sum_{n=1}^{\infty} \frac{b_{n}}{z^{n}}
$$

be a function belonging to $\Sigma^{\prime}$ and let

$$
G(z)=z+\sum_{n=1}^{\infty} \frac{c_{n}}{z^{n}}
$$


be the inverse function of $g(z)$, then by a simple calculation we have

$$
\begin{aligned}
c_{5}= & -\left(b_{5}+4 b_{1} b_{3}+2 b_{2}{ }^{2}+2 b_{1}{ }^{3}\right), \\
c_{7}= & -\left(b_{7}+6 b_{1} b_{5}+6 b_{2} b_{4}+15 b_{1}{ }^{2} b_{3}+3 b_{3}{ }^{2}+15 b_{1} b_{2}{ }^{2}+5 b_{1}{ }^{4}\right), \\
c_{9}= & -\left(b_{9}+8 b_{1} b_{7}+8 b_{2} b_{6}+8 b_{3} b_{5}+4 b_{4}{ }^{2}+28 b_{1}{ }^{2} b_{5}+56 b_{1} b_{2} b_{4}+28 b_{1} b_{3}{ }^{2}+56 b_{1}{ }^{3} b_{3}\right. \\
& \left.+28 b_{2}{ }^{2} b_{3}+84 b_{1}{ }^{2} b_{2}{ }^{2}+14 b_{1}{ }^{5}\right) .
\end{aligned}
$$

In this paper we shall use the following notations:

$$
\begin{aligned}
& b_{1}=p+\imath x^{\prime}=1-x+i x^{\prime}, \\
& b_{2}=y+i y^{\prime}, \\
& b_{3}=\eta+\imath \eta^{\prime}, \\
& b_{4}=\xi+i \xi^{\prime}, \\
& b_{5}=\varphi+i \varphi^{\prime}, \\
& b_{6}=\phi+i \phi^{\prime} .
\end{aligned}
$$

3. Firstly we are concerned with the case $n=3$. By Grunsky's inequality with $N=3, x_{1}=x_{2}=0, x_{3}=1$ we have

$$
\left|b_{5}+b_{1} b_{3}+b_{2}^{2}+\frac{1}{3} b_{1}^{3}\right| \leqq \frac{1}{3} .
$$

Hence we have

$$
\left|c_{5}\right|=\left|b_{5}+4 b_{1} b_{3}+2{b_{2}}^{2}+2 b_{1}{ }^{3}\right| \leqq\left|3 b_{1} b_{3}+b_{2}{ }^{2}+\frac{5}{3} b_{1}{ }^{3}\right|+\frac{1}{3} .
$$

We put

$$
F=R\left(3 b_{1} b_{3}+b_{2}{ }^{2}+\frac{5}{3} b_{1}{ }^{3}\right)+\frac{1}{3} .
$$

Since the polynomial $3 b_{1} b_{3}+b_{2}{ }^{2}+(5 / 3) b_{1}{ }^{3}$ is homogeneous, it is sufficient to prove that $F \leqq 2$ for $\left|\arg b_{1}\right| \leqq(\pi / 3)$. Rewriting (2) with the notations (1) we have

$$
F=2-5 x+5 x^{2}-\frac{5}{3}-x^{3}+3 p \eta+y^{2}-5 p x^{\prime 2}-y^{\prime 2}-3 x^{\prime} \eta^{\prime} .
$$

And it is evident that $0 \leqq p \leqq 1$ and $x^{\prime 2} \leqq 3 p^{2}$ when $\left|\arg b_{1}\right| \leqq(\pi / 3)$. By Grunsky's inequality with $N=2, x_{1}=0, x_{2}=1$ we have

$$
\left|b_{3}+\frac{1}{2} b_{1}^{2}\right| \leqq \frac{1}{2} \text {. }
$$

By taking the real part we have

$$
\eta \leqq x-\frac{1}{2} x^{2}+\frac{1}{2} x^{\prime 2} .
$$


Hence we have

$$
F \leqq 2-2 x+\frac{1}{2} x^{2}-\frac{1}{6} x^{3}+y^{2}-\frac{7}{2} p x^{\prime 2}-y^{\prime 2}-3 x^{\prime} \eta^{\prime} .
$$

By the area theorem

$$
-2 x+x^{2} \leqq-x^{\prime 2}-2 y^{2}-3 \eta^{\prime 2}
$$

Thus we obtain

$$
F \leqq 2-\frac{1}{2} x^{2}\left(1+\frac{1}{3} x\right)-y^{2}-y^{\prime 2}-\left(1+\frac{7}{2} p\right) x^{\prime 2}-3 \eta^{\prime 2}-3 x^{\prime} \eta^{\prime} .
$$

Since $0 \leqq p \leqq 1$,

$$
-\left(1+\frac{7}{2} p\right) x^{\prime 2}-3 x^{\prime} \eta^{\prime}-3 \eta^{\prime 2} \leqq 0 .
$$

Therefore (3) implies the desired result:

$$
F \leqq 2 \text {. }
$$

Equality occurs only for $x=0$.

4. Next we consider the case $n=4$. By Grunsky's inequality with $N=4$, $x_{1}=8 b_{2}, x_{2}=5 b_{1}, x_{3}=0, x_{4}=1$ we have

$$
\begin{aligned}
& \left|b_{7}+6 b_{1} b_{5}+6 b_{2} b_{4}+\frac{49}{4} b_{1}{ }^{2} b_{3}+\frac{3}{2} b_{3}{ }^{2}+-\frac{37}{2} b_{1} b_{2}{ }^{2}+\frac{27}{8}-b_{1}{ }^{4}\right| \\
& \leqq 4\left|b_{2}\right|^{2}+\frac{25}{8}\left|b_{1}\right|^{2}+\frac{1}{4} \text {. }
\end{aligned}
$$

Hence we have

$$
\begin{aligned}
\left|c_{7}\right|= & \left|b_{7}+6 b_{1} b_{5}+6 b_{2} b_{4}+15 b_{1}{ }^{2} b_{3}+3 b_{3}{ }^{2}+15 b_{1} b_{2}{ }^{2}+5 b_{1}{ }^{4}\right| \\
\leqq & \left|\frac{11}{4} b_{1}{ }^{2} b_{3}+\frac{3}{2} b_{3}{ }^{2}-\frac{7}{2} b_{1} b_{2}{ }^{2}+\frac{13}{8} b_{1}{ }^{4}\right| \\
& +4\left|b_{2}\right|^{2}+\frac{25}{8}\left|b_{1}\right|^{2}+\frac{1}{4} .
\end{aligned}
$$

Further by using Grunsky's inequality with $N=2 ; x_{1}=0, x_{2}=1$

we have

$$
\left|b_{3}+\frac{1}{2} b_{1}^{2}\right| \leqq \frac{1}{2} \text {. }
$$

$$
\left|c_{7}\right| \leqq\left|\frac{3}{2} b_{3}{ }^{2}-\frac{7}{2} b_{1} b_{2}{ }^{2}+\frac{1}{4} b_{1}{ }^{4}\right|+4\left|b_{2}\right|^{2}+\frac{9}{2}\left|b_{1}\right|^{2}+\frac{1}{4} .
$$

We put

$$
F=\mathscr{R}\left(\frac{3}{2} b_{3}{ }^{2}-\frac{7}{2} b_{1} b_{2}{ }^{2}+\frac{1}{4} b_{1}{ }^{4}\right)+4\left|b_{2}\right|^{2}+\frac{9}{2}\left|b_{1}\right|^{2}+\frac{1}{4} .
$$


Now it is sufficient to prove that $F \leqq 5$ for $\left|\arg b_{1}\right| \leqq(\pi / 4)$. Rewriting (4) with the notations (1) we have

$$
F=5-10 x+6 x^{2}-x^{3}+\frac{1}{4} x^{4}+\left(4-\frac{7}{2} p\right) y_{2}+\frac{3}{2} \eta^{2}
$$

$$
+\left(\frac{9}{2}-\frac{3}{2} p^{2}+\frac{1}{4} x^{\prime 2}\right) x^{\prime 2}+\left(4+\frac{7}{2} p\right) y^{\prime 2}-\frac{3}{2} \eta^{\prime 2}+7 x^{\prime} y^{\prime} y \text {. }
$$

And it is evident that $0 \leqq p \leqq 1$ and $x^{\prime 2} \leqq p^{2}$ when $\left|\arg b_{1}\right| \leqq(\pi / 4)$. By the area theorem

$$
-9 x+\frac{9}{2} x^{2} \leqq-\frac{9}{2} x^{\prime 2}-9 y^{2}-9 y^{\prime 2}-\frac{27}{2} \eta^{2}-\frac{27}{2} \eta^{\prime 2} .
$$

Putting (6) into (5), we obtain

$$
F \leqq 5-x P(x)-\left(5+\frac{7}{2} p\right) y^{2}-12 \eta^{2}-\left(\frac{3}{2} p^{2}-\frac{1}{4} x^{\prime 2}\right) x^{\prime 2}
$$

$$
-\left(5-\frac{7}{2} p\right) y^{\prime 2}-15 \eta^{\prime 2}+7 x^{\prime} y^{\prime} y
$$

$$
P(x)=1-\frac{3}{2} x+x^{2}-\frac{1}{4} x^{3} .
$$

Since $x^{\prime 2} \leqq p^{2} \leqq 1$, we have

$$
-\left(5+\frac{7}{2} p\right) y^{2}+7 x^{\prime} y^{\prime} y-\left(5-\frac{7}{2} p\right) y^{\prime 2} \leqq 0 \text {. }
$$

It is easy to prove that $P(x)>0$ for $0 \leqq p \leqq 1$. Therefore (7) implies that $F \leqq 5$ for $\left|\arg b_{1}\right| \leqq(\pi / 4)$, with equality holding only for $x=0$.

5. Finally we are concerned with the case $n=5$. By Grunsky's inequality with $N=5, x_{1}=0, x_{2}=10 b_{2}, x_{3}=(35 / 6) b_{1}, x_{4}=0, x_{5}=1$ we have

$$
\begin{aligned}
\mid b_{9}+8 b_{1} b_{7}+10 b_{2} b_{6}+3 b_{3} b_{5}+2 b_{4}{ }^{2} & +\frac{81}{4} b_{1}{ }^{2} b_{5}+54 b_{1} b_{2} b_{4}+10 b_{1} b_{3}{ }^{2}+28 b_{2}{ }^{2} b_{3} \\
& +\frac{81}{4} b_{1}{ }^{3} b_{3}+\frac{233}{4} b_{1}{ }^{2} b_{2}{ }^{2}+\frac{257}{60} b_{1}{ }^{5} \mid \\
& \leqq 8\left|b_{2}\right|^{2}+\frac{49}{12}\left|b_{1}\right|^{2}+\frac{1}{5} .
\end{aligned}
$$

Hence we have

$$
\begin{array}{r}
\left|c_{9}\right| \leqq \mid b_{9}+8 b_{1} b_{7}+8 b_{2} b_{6}+8 b_{3} b_{5}+4 b_{4}{ }^{2}+28 b_{1}{ }^{2} b_{5}+56 b_{1} b_{2} b_{4}+28 b_{1} b_{3}{ }^{2} \\
+28 b_{2}{ }^{2} b_{3}+56 b_{1}{ }^{3} b_{3}+84 b_{1}{ }^{2} b_{2}{ }^{2}+14 b_{1}{ }^{5} \mid
\end{array}
$$




$$
\begin{aligned}
& \leqq-2 b_{2} b_{6}+5 b_{3} b_{5}+2 b_{4}{ }^{2}+\frac{31}{4} b_{1}{ }^{2} b_{5}+2 b_{1} b_{2} b_{4} \\
& +18 b_{1} b_{3}{ }^{2}+\frac{143}{4} b_{1}{ }^{3} b_{3}+\frac{103}{4} b_{1}{ }^{2}{b_{2}}{ }^{2}+\frac{583}{60} b_{1}{ }^{5} \mid \\
& +8\left|b_{2}\right|_{2}+\frac{49}{12}\left|b_{1}\right|^{2}+\frac{1}{5} \text {. }
\end{aligned}
$$

Further by using Grunsky's inequality with $N=3, x_{1}=x_{2}=0, x_{3}=1$

$$
\left|b_{5}+b_{1} b_{3}+b_{2}^{2}+\frac{1}{3} b_{1}^{3}\right| \leqq \frac{1}{3},
$$

we have

$$
\begin{aligned}
\left|c_{9}\right| \leqq & \left|-2 b_{2} b_{6}+5 b_{3} b_{5}+2 b_{4}{ }^{2}+2 b_{1} b_{2} b_{4}+18 b_{1} b_{3}{ }^{2}+28 b_{1}{ }^{3} b_{3}+18 b_{1}{ }^{2} b_{2}{ }^{2}+\frac{107}{15} b_{1}{ }^{5}\right| \\
& +8\left|b_{2}\right|^{2}+\frac{20}{3}\left|b_{1}\right|^{2}+\frac{1}{5} .
\end{aligned}
$$

We put

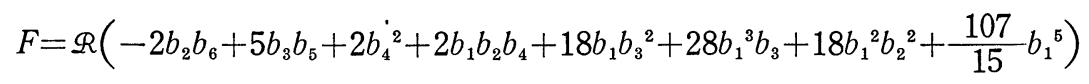

$$
+8\left|b_{2}\right|^{2}+\frac{20}{3}\left|b_{1}\right|^{2}+\frac{1}{5} .
$$

Now it is sufficient to prove that $F \leqq 14$ for $\left|\arg b_{1}\right| \leqq(\pi / 5)$. Rewriting (8) with the notations (1) we have

$$
\begin{aligned}
F= & 14-49 x+78 x^{2}-\frac{214}{3} x^{3}+\frac{107}{3} x^{4}-\frac{107}{15} x^{5}+28 p^{3} \eta \\
& +\left(8+18 p^{2}-18 x^{\prime 2}\right) y^{2}+18 p \eta^{2}+2 \xi^{2}+2 p y \xi-2 y \phi+5 \eta \varphi \\
& +\left(\frac{20}{3}-\frac{214}{3} p^{3}+\frac{107}{3} p x^{\prime 2}\right) x^{\prime 2}+\left(8-18 p^{2}+18 x^{\prime 2}\right) y^{\prime 2}-18 p \eta^{\prime 2} \\
& -2 \xi^{\prime 2}+\left(-84 p^{2}+28 x^{\prime 2}\right) x^{\prime} \eta^{\prime}-2 p y^{\prime} \xi^{\prime}+2 y^{\prime} \phi^{\prime}-5 \eta^{\prime} \varphi^{\prime} \\
& +y\left(-72 p x^{\prime} y^{\prime}-2 x^{\prime} \xi^{\prime}\right)+\eta\left(-84 p x^{\prime 2}-36 x^{\prime} \eta^{\prime}\right)-2 x^{\prime} y^{\prime} \xi .
\end{aligned}
$$

And it is evident that $0 \leqq p \leqq 1$ and $x^{\prime 2}<0.53 p^{2}$ when $\left|\arg b_{1}\right| \leqq(\pi / 5)$. Here we make use of Golusin's inequality. We put $N=2, x_{1}=0, x_{2}=1$ in Golusin's inequ. ality. Then we have

$$
\left|2 b_{2}\right|^{2}+2\left|2 b_{3}+b_{1}{ }^{2}\right|^{2} \leqq 2
$$

Rewriting this we have

$$
\begin{aligned}
4 p^{2} \eta \leqq & 4 x-6 x^{2}+4 x^{3}-x^{4}-2 y^{2}-4 \eta^{2}-\left(2 p^{2}+x^{\prime 2}\right) x^{\prime 2}-2 y^{\prime 2}-4 \eta^{\prime 2} \\
& -8 p x^{\prime} \eta^{\prime}+4 x^{\prime 2} \eta
\end{aligned}
$$


Putting (10) into (9), we have

$$
\begin{aligned}
F \leqq & 14-21 x+8 x^{2}-\frac{4}{3} x^{3}+\frac{2}{3} x^{4}-\frac{2}{15} x^{5} \\
& +\left(8-14 p+18 p^{2}-18 x^{\prime 2}\right) y^{2}-10 p \eta^{2}+2 \xi^{2}+2 p y \xi-2 y \phi+5 \eta \varphi \\
& +\left(\frac{20}{3}-\frac{256}{3} p^{3}+\frac{86}{3} p x^{\prime 2}\right) x^{\prime 2}+\left(8-14 p-18 p^{2}+18 x^{\prime 2}\right) y^{\prime 2} \\
& -46 p \eta^{\prime 2}-2 \xi^{\prime 2}+\left(-140 p^{2}+28 x^{\prime 2}\right) x^{\prime} \eta^{\prime}-2 p y^{\prime} \xi^{\prime}+2 y^{\prime} \phi^{\prime}-5 \eta^{\prime} \varphi^{\prime} \\
& +y\left(-72 p x^{\prime} y^{\prime}-2 x^{\prime} \xi^{\prime}\right)+\eta\left(-56 p x^{\prime 2}-36 x^{\prime} \eta^{\prime}\right)-2 x^{\prime} y^{\prime} \xi .
\end{aligned}
$$

By the area theorem

$$
\begin{aligned}
-21 x+\frac{21}{2} x^{2} \leqq & -\frac{21}{2} x^{\prime 2}-21 y^{2}-21 y^{\prime 2}-\frac{63}{2} \eta^{2}-\frac{63}{2} \eta^{\prime 2} \\
& -42 \xi^{2}-42 \xi^{\prime 2}-\frac{105}{2} \varphi^{2}-\frac{105}{2} \varphi^{\prime 2}-63 \phi^{2}-63 \phi^{\prime 2} .
\end{aligned}
$$

Hence we obtain

$$
\begin{aligned}
F \leqq & 14-x^{2} P(x)-Q, \\
P(x)= & \frac{5}{2}+\frac{4}{3} x-\frac{2}{3} x^{2}+\frac{2}{15} x^{3}, \\
Q= & \left(13+14 p-18 p^{2}+18 x^{\prime 2}\right) y^{2}+(31.5+10 p) \eta^{2}+40 \xi^{2}+52.5 \varphi^{2} \\
& +63 \phi^{2}-2 p y \xi+2 y \phi-2 \cdot 2.5 \eta \varphi \\
& +\left(3.833+85.333 p^{3}-28.667 p x^{\prime 2}\right) x^{\prime 2}+\left(13+14 p+18 p^{2}-18 x^{\prime 2}\right) y^{\prime 2} \\
& +(31.5+46 p) \eta^{\prime 2}+44 \xi^{\prime 2}+52.5 \varphi^{\prime 2}+63 \phi^{\prime 2}+2\left(70 p^{2}-14 x^{\prime 2}\right) x^{\prime} \eta^{\prime} \\
& +2 p y^{\prime} \xi^{\prime}-2 y^{\prime} \phi^{\prime}+2 \cdot 2.5 \eta^{\prime} \varphi^{\prime} \\
& +2 \cdot 36 p x^{\prime} y^{\prime} y+2 x^{\prime} \xi^{\prime} y+2 \cdot 28 p x^{\prime 2} \eta+2 \cdot 18 x^{\prime} \eta^{\prime} \eta+2 x^{\prime} y^{\prime} \xi .
\end{aligned}
$$

It is evident that $P(x)>0$ for $0 \leqq p \leqq 1$. In order to prove $Q \geqq 0$, we first observe

$$
\begin{aligned}
& 0.12 \eta^{2}-2 \cdot 2.5 \eta \varphi+52.5 \varphi^{2} \geqq 0, \\
& 0.12 \eta^{\prime 2}+2 \cdot 2.5 \eta^{\prime} \varphi^{\prime}+52.5 \varphi^{\prime 2} \geqq 0, \\
& 12 \eta^{2}+2 \cdot 18 x^{\prime} \eta^{\prime} \eta+27 x^{\prime 2} \eta^{\prime 2} \geqq 0, \\
& (19.38+10 p) \eta^{2}+2 \cdot 28 p x^{\prime 2} \eta+26.685 p x^{\prime 4} \geqq 0 .
\end{aligned}
$$

Further we have

$$
\begin{aligned}
\left(3.833+85.333 p^{3}-55.352 p x^{\prime 2}\right) x^{\prime 2}+ & 2\left(70 p^{2}-14 x^{\prime 2}\right) x^{\prime} \eta^{\prime} \\
& +\left(31.38+46 p-27 x^{\prime 2}\right) \eta^{\prime 2} \geqq 0 .
\end{aligned}
$$


Indeed we consider the discriminant $\Delta$ of this quadratic from. Then

$$
\begin{aligned}
\Delta> & 120.279+176.318 p+2677.749 p^{3}-974.682 p^{4} \\
& -\left(103.491+1736.946 p+586.192 p^{2}+2303.991 p^{3}\right) x^{\prime 2} \\
& +(1494.504 p-196) x^{\prime 4} .
\end{aligned}
$$

If $x^{\prime 2} \leqq 0.45 p^{2}$ and $0 \leqq p \leqq 1$, then

$$
\begin{aligned}
\Delta> & 120.279+176.318 p+2677.749 p^{3}-947.682 p^{4} \\
& -0.45 p^{2}\left(103.491+1736.946 p+586.192 p^{2}+2303.991 p^{3}\right) \\
& +(0.45)^{2} p^{4}(1494.504 p-196) \\
\geqq & 120.279+176.318 p-46.571 p^{2} \\
& +1896.123 p^{3}-1278.159 p^{4}-734.159 p^{5}>0 .
\end{aligned}
$$

If $0.45 p^{2} \leqq x^{\prime 2}<0.53 p^{2}$, then $0 \leqq p<0.84$, whence

$$
\Delta>120.279+176.318 p-54.851 p^{2}+1757.167 p^{3}-1340.421 p^{4}-801.31 p^{5}>0 .
$$

Thus we have the desired inequality. By using these inequalities we have

$$
\begin{aligned}
Q & \geqq\left(13+14 p-18 p^{2}+18 x^{\prime 2}\right) y^{2}+40 \xi^{2}+63 \phi^{2}-2 p y \xi+2 y \phi \\
& +\left(13+14 p+18 p^{2}-18 x^{2}\right) y^{\prime 2}+44 \xi^{\prime 2}+63 \phi^{\prime 2}+2 p y^{\prime} \xi^{\prime}-2 y^{\prime} \phi^{\prime} \\
& +2 \cdot 36 p x^{\prime} y^{\prime} y+2 x^{\prime} \xi^{\prime} y+2 x^{\prime} y^{\prime} \xi .
\end{aligned}
$$

Further by using the inequalities

we have

$$
\begin{aligned}
& \xi^{2}+2 x^{\prime} y^{\prime} \xi+x^{\prime 2} y^{\prime 2} \geqq 0, \\
& 4 \xi^{\prime 2}+2 x^{\prime} \xi^{\prime} y+0.25 x^{\prime 2} y^{2} \geqq 0, \\
& 36 x^{\prime 2} y^{2}+2 \cdot 36 p x^{\prime} y^{\prime} y+36 p^{2} y^{\prime 2} \geqq 0,
\end{aligned}
$$

$$
\begin{aligned}
Q \geqq & \left(13+14 p-18 p^{2}-18.25 x^{\prime 2}\right) y^{2}+39 \xi^{2}+63 \phi^{2}-2 p y \xi+2 y \phi \\
& +\left(13+14 p-18 p^{2}-19 x^{\prime 2}\right) y^{\prime 2}+40 \xi^{\prime 2}+63 \phi^{\prime 2}+2 p y^{\prime} \xi^{\prime}-2 y^{\prime} \phi^{\prime} .
\end{aligned}
$$

We consider the symmetric matrix associated with the quadratic form

$$
\begin{gathered}
\left(13+14 p-18 p^{2}-18.25 x^{\prime 2}\right) y^{2}+39 \xi^{2}+63 \phi^{2}-2 p y \xi+2 y \phi: \\
\left(\begin{array}{rrc}
63 & 0 & 1 \\
0 & 39 & -p \\
1 & -p & 13+14 p-18 p^{2}-18.25 x^{\prime 2}
\end{array}\right) .
\end{gathered}
$$

Its principal diagonal minor determinants are 
63 ,

2457 ,

$31902+34398 p-44289 p^{2}-44840.25 x^{\prime 2} \equiv \Delta$.

If $x^{\prime 2} \leqq 0.45 p^{2}$ and $0 \leqq p \leqq 1$, then

$$
\Delta \geqq 31902+34398 p-64468 p^{2}>0 .
$$

If $0.45 p^{2} \leqq x^{\prime 2}<0.53 p^{2}$ and $0 \leqq p<0.84$, then

$$
\Delta \geqq 31902+34398 p-68055 p^{2}>0 \text {. }
$$

Hence it follows that $\left(13+14 p-18 p^{2}-18.25 x^{\prime 2}\right) y^{2}+39 \xi^{2}+63 \phi^{2}-2 p y \xi+2 y \phi \geqq 0$ for $\left|\arg b_{1}\right| \leqq(\pi / 5)$. Similarly it follows that $\left(13+14 p-18 p^{2}-19 x^{\prime 2}\right) y^{\prime 2}+40 \xi^{\prime 2}+63 \phi^{\prime 2}+$ $2 \not y^{\prime} \xi^{\prime}-2 y^{\prime} \phi^{\prime} \geqq 0$ for $\left|\arg b_{1}\right| \leqq(\pi / 5)$. Consequently we have $Q \geqq 0$. Thus (11) implies that $F \leqq 14$ for $\left|\arg b_{1}\right| \leqq(\pi / 5)$, with equality holding only for $x=0$.

\section{REFERENCES}

[1] Golusin, G. M., On p-valent functions. Mat. Sbornik (N.S.) 8 (1940), 277-284.

[2] GRunsky, H., Koeffizientenbedingungen für schlicht abbildende meromorphe Funktionen. Math. Zeits. 4 (1939), 29-61.

[3] Ozawa, M., On the Bieberbach conjecture for the sixth coefficient. Ködai Math. Sem. Rep. 21 (1969), 97-128.

[4] SPRINGER, G., The coefficient problem for schlicht mappings of the exterior of the unit circle. Trans. Amer. Math. Soc. 70 (1951), 421-450.

Tokyo Gakugei University. 